Article

\title{
LED Nonlinearity Estimation and Compensation in VLC Systems Using Probabilistic Bayesian Learning
}

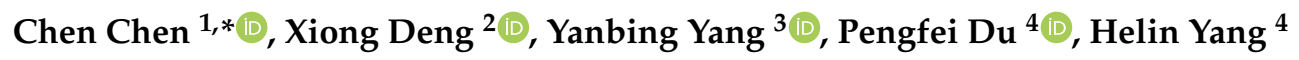 \\ and Lifan Zhao ${ }^{4}$ \\ 1 School of Microelectronics and Communication Engineering, Chongqing University, \\ Chongqing 400044, China \\ 2 Department of Electrical Engineering, Eindhoven University of Technology (TU/e), Flux Building, \\ 5600MB Eindhoven, The Netherlands \\ 3 College of Computer Science, Sichuan University, Chengdu 610065, China \\ 4 School of Electrical and Electronic Engineering, Nanyang Technological University, \\ Singapore 639798, Singapore \\ * Correspondence: c.chen@cqu.edu.cn
}

Received: 27 May 2019; Accepted: 29 June 2019; Published: 3 July 2019

\begin{abstract}
In this paper, we propose and evaluate a novel light-emitting diode (LED) nonlinearity estimation and compensation scheme using probabilistic Bayesian learning (PBL) for spectral-efficient visible light communication (VLC) systems. The nonlinear power-current curve of the LED transmitter can be accurately estimated by exploiting PBL regression and hence the adverse effect of LED nonlinearity can be efficiently compensated. Simulation results show that, in a 80-Mbit/s orthogonal frequency division multiplexing (OFDM)-based nonlinear VLC system, comparable bit-error rate (BER) performance can be achieved by the conventional time domain averaging (TDA)-based LED nonlinearity mitigation scheme with totally 20 training symbols (TSs) and the proposed PBL-based scheme with only a single TS. Therefore, compared with the conventional TDA scheme, the proposed PBL-based scheme can substantially reduce the required training overhead and hence greatly improve the overall spectral efficiency of bandlimited VLC systems. It is also shown that the PBL-based LED nonlinearity estimation and compensation scheme is computational efficient for the implementation in practical VLC systems.
\end{abstract}

Keywords: light emitting diode; nonlinearity estimation and compensation; probabilistic Bayesian learning; visible light communication

\section{Introduction}

Visible light communication (VLC) relying on white illuminating light-emitting diodes (LEDs) has attracted extensive interest in recent years, due to its inherent advantages such as unregulated spectrum, relatively low implementation cost, enhanced physical-layer security, and electromagnetic interference-free operation [1,2]. The emerging VLC technology has revealed great potential for a lot of practical applications such as high-speed communications, wireless networking, human sensing, ranging and detecting [3,4]. Nevertheless, white LEDs have several limitations which might greatly hinder the development and implementation of VLC systems in practical applications. One limitation is the small modulation bandwidth (typically a few $\mathrm{MHz}$ ) due to the physical mechanism in the LED quantum well [5] and the long photoluminescence lifetimes of the phosphor, resulting in inter-symbol interference [6]. Several techniques have already been reported for capacity improvement of VLC systems, such as spectral efficiency enhancement employing orthogonal frequency division 
multiplexing (OFDM) with high-order quadrature amplitude modulation (QAM) constellations [7] and non-orthogonal multiple access [8-11], multiple-input multiple-output (MIMO) transmission [12,13], bandwidth extension using various frequency-domain equalization schemes [14,15], and so on.

Another limitation is that white LEDs suffer from intrinsic nonlinearity which is mainly caused by the thermal effects. It has been shown that LEDs are the major source of nonlinearity in typical VLC systems [16]. Due to LED nonlinearity, the input signal could be severely distorted, especially for OFDM signals which usually have high peak-to-average power ratios (PAPRs) [17]. Generally, there are two approaches to mitigate LED nonlinearity: one is estimation and compensation at transmitter or receiver side and the other is nonlinear equalization. For the first approach, the nonlinear power-current curve of the LED is first estimated, which is then used to compensate the LED nonlinearity. In [18-20], transmitter-side LED nonlinearity estimation and compensation, i.e., pre-distortion, has been considered. In [21,22], receiver-side LED nonlinearity estimation and compensation, i.e., post-distortion, has been applied. For pre-distortion, the estimated nonlinear power-current curve of the LED is treated as a priori information and hence an additional feedback channel is required. In contrast, no feedback is required for post-distortion. For both pre-distortion and post-distortion, time domain averaging (TDA) is usually adopted for accurate estimation of the nonlinear power-current curve of the LED before LED nonlinearity compensation. Nevertheless, conventional TDA usually needs a relatively large number of training symbols (TSs) to achieve the expected performance, which inevitably reduce the spectral efficiency of VLC systems. For the second approach, LED nonlinearity is mitigated by employing various nonlinear equalizers, such as Volterra series-based equalizers [17,23], clustering-based equalizers [24,25], deep learning-based equalizers [26,27], and so on. However, nonlinear equalizers usually require a relatively large training overhead and also suffer from high computational complexity, which might not be suitable for implementation in practical VLC systems due to the limited computing capability of user terminals.

As a widely used machine learning technique, support vector machine (SVM) has already been applied for the mitigation of fiber nonlinearity in coherent optical OFDM systems [28]. However, SVM is a non-probabilistic machine which usually requires a large number of kernels to approximate the optimal solution and hence its application in practical systems is limited. Recently, probabilistic program induction was proposed, which can substantially improve the accuracy of machine learning algorithms when only a few examples are available [29]. In [30], a probabilistic Bayesian learning (PBL) framework was introduced which can obtain a similar generalization performance as that of SVM but needs much fewer basis functions. The PBL technique has many potential applications such as channel estimation [31], radar imagery [32] and frequency-hopping spectrum estimation [33].

In this paper, we for the first time propose a PBL-based LED nonlinearity estimation and compensation scheme for OFDM-based nonlinear VLC systems. The LED nonlinearity, i.e., nonlinear power-current curve, can be accurately estimated by PBL regression and hence the adverse effect of LED nonlinearity can be efficiently compensated at the receiver side. Numerical simulations are performed to validate the feasibility of the proposed PBL-based LED nonlinearity estimation and compensation scheme in a 80-Mbit/s OFDM-based nonlinear VLC system and performance comparison between the proposed PBL-based scheme and the conventional TDA scheme is provided. The computational complexity of the proposed PBL-based LED nonlinearity estimation and compensation scheme is also analyzed.

The rest of this paper is organized as follows. In Section 2, we first introduce the mathematical model of an OFDM-based nonlinear VLC system. Section 3 presents the principle of the proposed PBL-based LED nonlinearity estimation and compensation scheme. The simulation setup is described in Section 4 and the detailed results and discussions are provided in Section 5. Finally, Section 6 concludes the paper. 


\section{System Model}

In this section, we introduce the model of an OFDM-based nonlinear VLC system and the block diagram of the system model is illustrated in Figure 1. As we can see, the input bits are first modulated into real-valued OFDM symbols and then TSs are added for efficient LED nonlinearity estimation and compensation at the receiver side. The obtained digital signal is subsequently transformed into an analog signal through digital-to-analog conversion (DAC), and then a direct current (DC) bias is further added to convert the bipolar signal into a unipolar signal in order to generate a real-valued nonnegative driving signal for the LED transmitter. After that, the generated signal is fed into a white LED which suffers from nonlinearity. In order to support a long transmission distance and a large communication coverage area in typical indoor environments, a relatively high modulation index (MI) is generally required when modulating the signal to the LEDs in practical VLC systems. However, the input signal could be significantly distorted when using a high MI due to LED nonlinearity [34].

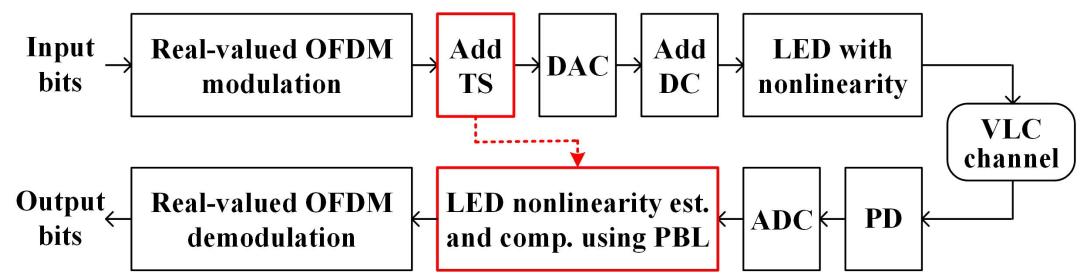

Figure 1. Block diagram of an OFDM-based nonlinear VLC system using PBL-based LED nonlinearity estimation (est.) and compensation (comp.).

The visible light radiated from the white LED propagates through the free-space VLC channel for simultaneous illumination and communication. For simplicity and without loss of generality, it is reasonable to only consider the line-of-sight (LOS) component in the system model [12]. Assuming that the LED follows a generalized Lambertian pattern, the LOS optical channel gain can be calculated by [1]

$$
h=\frac{(m+1) \rho A}{2 \pi d^{2}} \cos ^{m}(\varphi) G_{f} G_{l} \cos (\theta),
$$

where $m$ is the order of Lambertian emission which is given by $m=-\ln 2 / \ln (\cos (\Psi))$ with $\Psi$ being the semi-angle at half power of the LED transmitter; $\rho$ and $A$ are the responsivity and the active area of the photodetector (PD), respectively; $d$ is the distance between the LED and the PD; $\varphi$ and $\theta$ are the corresponding emission angle and incident angle, respectively; $G_{f}$ and $G_{l}$ are the gains of the optical filter and the optical lens, respectively. The gain of the optical lens is given by $G_{l}=\frac{n^{2}}{\sin ^{2} \Phi}$, where $n$ and $\Phi$ are the refractive index and the half-angle field-of-view (FOV) of the optical lens, respectively. Please note that the LOS channel gain becomes zero if the incident light is outside the FOV of the receiver.

At the receiver side, the light is detected by a PD and the obtained electrical OFDM signal can be expressed by [31]

$$
y(t)=P_{0} h \xi f_{x}(t)+n(t),
$$

where $P_{0}$ is the average output optical power of the LED, $h$ is the optical channel gain defined in Equation (1), $\xi$ is the MI of the LED, $f_{x}(t)$ is the distorted version of the transmitted OFDM signal $x(t)$ due to LED nonlinearity, and $n(t)$ is the additive white Gaussian noise (AWGN) including both shot and thermal noises. The detailed expressions of the noise variances can be found in [31]. The obtained analog OFDM signal is then converted to a digital signal through analog-to-digital conversion (ADC). In order to mitigate the adverse effect of LED nonlinearity in VLC systems, PBL-based LED nonlinearity estimation and compensation are subsequently executed. The detailed procedures of LED nonlinearity estimation and compensation using PBL are described in the next section. After that, the compensated OFDM signal is achieved which is further demodulated to generate the output bits. The principle of real-valued OFDM modulation/demodulation can be found in [31], which is omitted here for brevity. 


\section{PBL-Based LED Nonlinearity Estimation and Compensation in VLC}

\subsection{PBL Regression}

Following the PBL regression model described in [30,35], for a given data set of input-target pairs $\left\{\boldsymbol{s}_{n}, \tau_{n}\right\}_{n=1}^{N}$ with length $N$, the target samples $\left\{\tau_{n}\right\}_{n=1}^{N}$ can be predicted by a linear combination of basis functions:

$$
\tau_{n}=\sum_{m=1}^{M} \mu_{m} \phi_{m}\left(s_{n}\right)+\epsilon_{n}=\boldsymbol{\mu}^{T} \boldsymbol{\phi}\left(\boldsymbol{s}_{n}\right)+\epsilon_{n}
$$

where $\boldsymbol{\mu}=\left[\mu_{1}, \mu_{2}, \cdots, \mu_{M}\right]^{T}$ is the parameter vector with length $M,\left\{\phi_{m}\left(\boldsymbol{s}_{n}\right)\right\}_{m=1}^{M}$ is a set of $M$ basis functions and the basis vector is expressed by $\phi\left(s_{n}\right)=\left[\phi_{1}\left(s_{n}\right), \phi_{2}\left(s_{n}\right), \cdots, \phi_{M}\left(s_{n}\right)\right]^{T}$, and $\epsilon=\left[\epsilon_{1}, \epsilon_{2}, \cdots, \epsilon_{N}\right]^{T}$ is the error vector due to the additive noise in the VLC system. Assuming that the error samples $\left\{\epsilon_{n}\right\}_{n=1}^{N}$ are independently and identically distributed Gaussian with zero mean and variance $\sigma^{2}$, a multivariate Gaussian likelihood for the target vector $\tau=\left[\tau_{1}, \tau_{2}, \cdots, \tau_{N}\right]^{T}$ can be written as follows [30]

$$
p\left(\boldsymbol{\tau} \mid \mu, \sigma^{2}\right)=\left(2 \pi \sigma^{2}\right)^{-N / 2} \exp \left(-\frac{\|\boldsymbol{\tau}-\boldsymbol{\Phi}(s) \boldsymbol{\mu}\|^{2}}{2 \sigma^{2}}\right),
$$

where $\boldsymbol{\Phi}(\boldsymbol{s})=\left[\boldsymbol{\phi}\left(\boldsymbol{s}_{1}\right), \boldsymbol{\phi}\left(\boldsymbol{s}_{2}\right), \cdots, \boldsymbol{\phi}\left(\boldsymbol{s}_{N}\right)\right]^{T}$ is an $N \times(N+1)$ design matrix with $M=N+1$. According to [30], the basis vector with a bias is defined as $\boldsymbol{\phi}\left(s_{n}\right)=\left[1, K\left(s_{n}, s_{1}\right), K\left(s_{n}, s_{2}\right), \cdots, K\left(s_{n}, s_{N}\right)\right]^{T}$, in which $K\left(s_{i}, s_{j}\right)$ is the kernel function. In this work, the Gaussian kernel is adopted and hence the kernel function is given by $K\left(s_{i}, s_{j}\right)=\exp \left(-\lambda\left\|s_{i}-s_{j}\right\|^{2}\right)$, where $\lambda$ is known as the width parameter.

From the Bayesian perspective, we can constrain the parameters by defining a zero-mean Gaussian prior distribution over them which takes the form:

$$
p(\boldsymbol{\mu} \mid \boldsymbol{\alpha})=\prod_{n=0}^{N} \mathcal{N}\left(\mu_{n} \mid 0, \alpha_{n}^{-1}\right),
$$

where $\alpha=\left[\alpha_{0}, \alpha_{1}, \alpha_{2}, \cdots, \alpha_{N}\right]^{T}$ is a vector of $N+1$ independent hyperparameters and each one is used to individually control the strength of the prior over its associated parameter [30]. By combining the likelihood and the prior within Bayes' rule, the posterior parameter distribution conditioned on $\tau$ can be obtained by

$$
p\left(\boldsymbol{\mu} \mid \boldsymbol{\tau}, \boldsymbol{\alpha}, \sigma^{2}\right)=\frac{p\left(\boldsymbol{\tau} \mid \boldsymbol{\mu}, \sigma^{2}\right) p(\boldsymbol{\mu} \mid \boldsymbol{\alpha})}{p\left(\boldsymbol{\tau} \mid \boldsymbol{\alpha}, \sigma^{2}\right)}
$$

which is Gaussian $\mathcal{N}(\boldsymbol{w}, \boldsymbol{\Sigma})$ and

$$
\begin{gathered}
\boldsymbol{\Sigma}=\left(\sigma^{-2} \boldsymbol{\Phi}^{T} \boldsymbol{\Phi}+\operatorname{diag}(\boldsymbol{\alpha})\right)^{-1}, \\
\boldsymbol{w}=\sigma^{-2} \boldsymbol{\Sigma} \boldsymbol{\Phi}^{T} \boldsymbol{\tau} .
\end{gathered}
$$

Since it is analytically intractable to include Bayesian inference over those hyperparameters, a type-II maximum likelihood procedure can be used to find a most-probable point estimate $\alpha_{\text {MP }}$ [30]. Therefore, PBL is formulated as the local maximization with respect to $\alpha$ of the marginal likelihood $p\left(\boldsymbol{\tau} \mid \boldsymbol{\alpha}, \sigma^{2}\right)$ and the logarithm of the marginal likelihood is given by [35]

$$
\begin{aligned}
\log \left(p\left(\boldsymbol{\tau} \mid \boldsymbol{\alpha}, \sigma^{2}\right)\right) & =\log \int_{-\infty}^{\infty} p\left(\boldsymbol{\tau} \mid \boldsymbol{\mu}, \sigma^{2}\right) p(\boldsymbol{\mu} \mid \boldsymbol{\alpha}) d \boldsymbol{\mu} \\
& =-\frac{1}{2}\left(N \log 2 \pi+\log |\boldsymbol{C}|+\boldsymbol{\tau}^{T} C^{-1} \boldsymbol{\tau}\right),
\end{aligned}
$$


where $\boldsymbol{C}=\sigma^{2} \boldsymbol{I}+\boldsymbol{\Phi}(\operatorname{diag}(\boldsymbol{\alpha}))^{-1} \boldsymbol{\Phi}^{T}$ and $\boldsymbol{I}$ is an identity matrix. Hence, a point estimate $\boldsymbol{w}_{\mathrm{MP}}$ for the weights can be obtained by evaluating Equation (8) with $\alpha=\alpha_{\mathrm{MP}}$. As a result, the final prediction of target $\tau$ is given by $\hat{\boldsymbol{\tau}}=\boldsymbol{\Phi}(s) w_{\mathrm{MP}}$.

\subsection{LED Nonlinearity Estimation and Compensation Using PBL Regression}

The principle of the proposed PBL-based LED nonlinearity estimation and compensation scheme is depicted in Figure 2. For simple and efficient LED nonlinearity (i.e., the nonlinear power-current curve of the LED) estimation, a sawtooth-based vector $z$ with $N$ samples, i.e., $z=\left[z_{1}, z_{2}, \cdots, z_{N}\right]^{T}$, is adopted as the TS for LED nonlinearity estimation using PBL regression in this work. The TS is known and shared by all the receivers within the coverage of the VLC system. After transmitting through the nonlinear VLC system, a corresponding vector $\boldsymbol{r}=\left[r_{1}, r_{2}, \cdots, r_{N}\right]^{T}$ can be detected at the receiver side, which can give the raw estimation of the LED nonlinearity. In order to obtain an accurate estimation of the LED nonlinearity, PBL regression is performed which takes the raw estimation of the LED nonlinearity $r$ as the input and the actual LED nonlinearity $\tau$ as the target. Although the design matrix is generated by using the input in [30,35], it has been found in our study that the sawtooth-based training vector $z$ can be directly used to generate the design matrix $\boldsymbol{\Phi}(z)$, which can achieve comparable performance as that using the input $r$. Taking $r$ as the input and using the design matrix $\boldsymbol{\Phi}(z)$, PBL regression can be successfully performed to obtain an accurate estimation of $\tau$, i.e., $\hat{\tau}$.

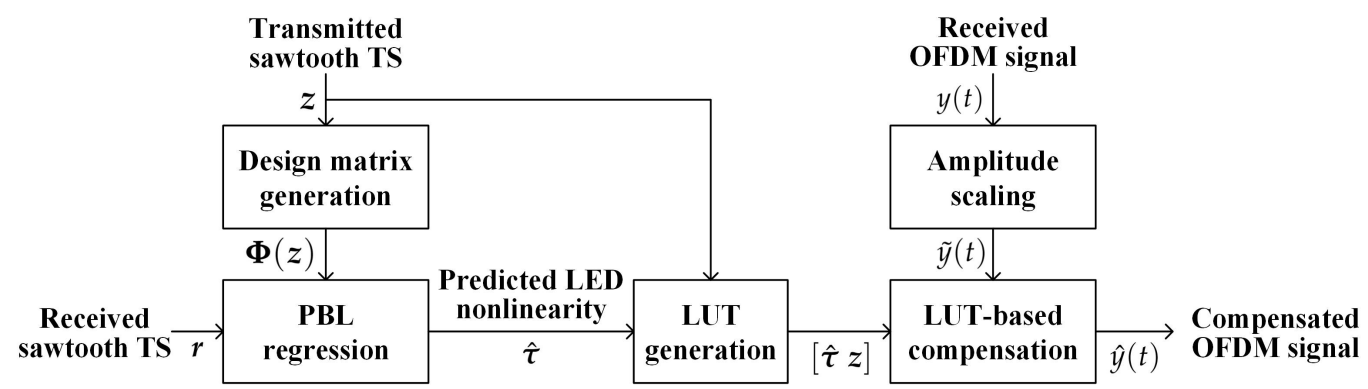

Figure 2. Principle of PBL-based LED nonlinearity estimation and compensation.

As shown in Figure 2, after obtaining $z$ and $\hat{\tau}$, a corresponding look-up table (LUT), i.e., $\mathcal{L}=[\hat{\boldsymbol{\tau}} z]$, can be generated. By using the generated LUT, LED nonlinearity compensation can be executed regarding the received OFDM signal. First, the amplitude of the received OFDM signal is scaled by a factor $P_{0} h \xi$ so as to match the amplitude of the transmitted OFDM signal, and hence the scaled OFDM signal is expressed by

$$
\tilde{y}(t)=\frac{y(t)}{P_{0} h \xi}=f_{x}(t)+\frac{n(t)}{P_{0} h \xi} .
$$

Then, using the obtained $\hat{\tau}=\left[\hat{\tau}_{1}, \hat{\tau}_{2}, \cdots, \hat{\tau}_{N}\right]^{T}$, the index $i(t)$ of the element in $\hat{\tau}$ which is closest to $\tilde{y}(t)$ can be identified as

$$
i(t)=\arg \min _{k}\left\{\tilde{y}(t)-\hat{\tau}_{k}\right\}, k \in\{1,2, \cdots, N\},
$$

and hence the compensated OFDM signal can be obtained by $\hat{y}(t)=\hat{\tau}_{i(t)}$.

\section{Simulation Setup}

Numerical simulations using MATLAB are performed to investigate the performance of the proposed PBL-based LED nonlinearity estimation and compensation scheme in an OFDM-based nonlinear VLC system. Key parameters of the simulation setup are listed in Table 1. The LED has a semi-angle at half power of $60^{\circ}$ and an output optical power of $10 \mathrm{~W}$. The gain of the optical filter is 0.9 . The refractive index and the half-angle FOV of the optical lens are 1.5 and $72^{\circ}$, respectively. 
The PD has an active area of $16 \mathrm{~mm}^{2}$ and a responsivity of $0.53 \mathrm{~A} / \mathrm{W}$. The vertical distance between LED and PD is set to $2 \mathrm{~m}$ and the horizontal offset between LED and PD is also assumed to be $2 \mathrm{~m}$. A modulation bandwidth of $20 \mathrm{MHz}$ is considered and 16QAM constellation is adopted in real-valued OFDM modulation/demodulation. Hence, the raw data rate of the OFDM-based nonlinear VLC system is $80 \mathrm{Mbit} / \mathrm{s}$. In OFDM modulation/demodulation, the size of fast Fourier transform (FFT)/inverse fast Fourier transform (IFFT) is set to 512. Due to the Hermitian symmetry constraint, only 128 subcarriers are used to carry valid data. A total of 1000 OFDM symbols are transmitted for bit-error rate (BER) calculation.

Table 1. Simulation parameters.

\begin{tabular}{cc}
\hline Parameter & Value \\
\hline Semi-angle at half power of LED & $60^{\circ}$ \\
LED output optical power & $10 \mathrm{~W}$ \\
Gain of optical filter & 0.9 \\
Refractive index of optical lens & 1.5 \\
Half-angle FOV of optical lens & $72^{\circ}$ \\
Active area of PD & $16 \mathrm{~mm}^{2}$ \\
Responsivity of PD & $0.53 \mathrm{~A} / \mathrm{W}$ \\
Vertical distance between LED and PD & $2 \mathrm{~m}$ \\
Horizontal offset between LED and PD & $2 \mathrm{~m}$ \\
Modulation bandwidth & $20 \mathrm{MHz}$ \\
QAM constellation order & 16 \\
Raw data rate & $80 \mathrm{Mbit} / \mathrm{s}$ \\
Size of FFT/IFFT & 512 \\
Number of data subcarriers & 128 \\
\hline
\end{tabular}

In the simulation setup, a commercially available white LED (Cree PLCC4) is used and the measured nonlinear power-current curve is illustrated in Figure 3. It can be observed that the normalized output optical power of the LED exhibits strong nonlinearity with the normalized input current. Since only unipolar signal can be modulated onto the luminous intensity, a DC bias current is generally applied to convert the bipolar OFDM signal to a unipolar one. Hence, the nonlinear distortion is mainly caused by two factors: the DC bias current and the peak-to-peak current of the signal. To investigate the LED nonlinearity effect on the transmission performance, as shown in Figure 3, we define the MI as the ratio of the maximum current variation of the signal to the maximum current variation supported by the LED without clipping, and the DC-bias index (DI) as the ratio of the DC bias current of the signal to the maximum current variation supported by the LED without clipping. In this simulation investigation, the LED is assumed to be biased at the middle point of its dynamic range, i.e., $\mathrm{DI}=0.5$. Although a static LED nonlinearity characteristic is considered in this work, the proposed PBL-based LED nonlinearity estimation and compensation scheme can be easily generalized into an adaptive scheme by adopting the method reported in [36], which is applicable in VLC systems with dynamic LED nonlinearity characteristics.

Moreover, the frequency selective fading effect of a VLC system is mainly caused by the LED, which can be easily compensated by using the frequency domain pre-equalization techniques [14,34]. Thus, a flat system frequency response is considered here without loss of generality. 


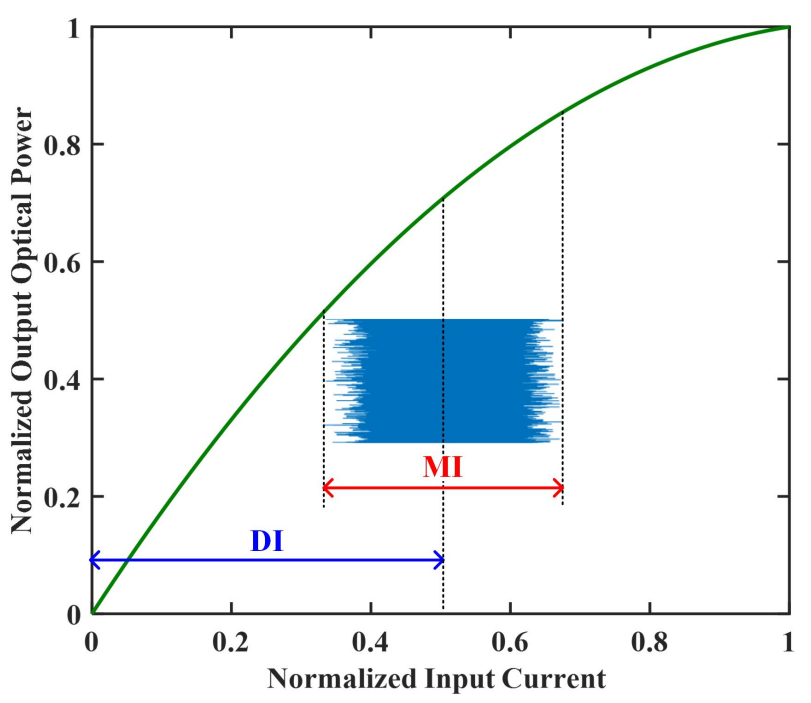

Figure 3. Measured nonlinear power-current curve of white LED Cree PLCC4.

The original PBL regression model proposed in [30] starts with all the $M=N+1$ basis functions. The update rules for the hyperparameters depend on computing the posterior weight co-variance matrix which requires a Cholesky decomposition operation with a complexity of $O\left(M^{3}\right)$. As a result, the computational complexity of PBL regression could be very high for practical applications. To reduce computational complexity, a fast marginal likelihood maximization method has been proposed in [35]. Based on an accelerated training algorithm, PBL regression is initialized with a single basis function, i.e., the bias. Sequentially, the basis functions are added to increase the marginal likelihood and also modify their weightings, and meanwhile the redundant basis functions are deleted to increase the objective function. Hence, the new PBL regression model can achieve comparable performance as the original one but with greatly reduced computational complexity, which is adopted here to realize efficient LED nonlinearity estimation and compensation in OFDM-based nonlinear VLC systems. Specifically, the width parameter of the Gaussian kernel is set to $\lambda=2$ and the maximum number of iterations is set to 10. For the purpose of comparison, the conventional TDA scheme employing multiple consecutive TSs is also considered in the simulations.

\section{Results and Discussion}

In this section, simulation results based on the setup described above are presented and the corresponding discussions are also provided. Figure 4 depicts the normalized signal amplitude vs. the normalized input current for different MI values, where DI $=0.5$ and the length of the sawtooth-based TS is $N=64$. As we can observe, for both $\mathrm{MI}=0.6$ and 0.8 , the amplitude of the received TS has a significant variation due to the additive noise in the VLC system. However, after performing PBL regression, the predicted power-current curve of the LED becomes much smoother which matches the actual power-current curve of the LED very well, indicating an accurate estimation of the LED nonlinearity by using only a single sawtooth-based TS.

We further analyze the impact of the number of TSs on the estimation accuracy of both the conventional TDA scheme and the proposed PBL-based scheme. Figure 5 shows the average estimation error vs. the number of TSs with $\mathrm{MI}=0.8, \mathrm{DI}=0.5$ and $N=64$. For the conventional TDA scheme, it can be seen that the average estimation error is gradually reduced with the increase of the number of TSs and it becomes stable at about $1.7 \times 10^{-3}$ when the number of TSs reaches 20 . In contrast, for the proposed PBL regression scheme, a comparable average estimation error can be achieved by using only a single TS. 

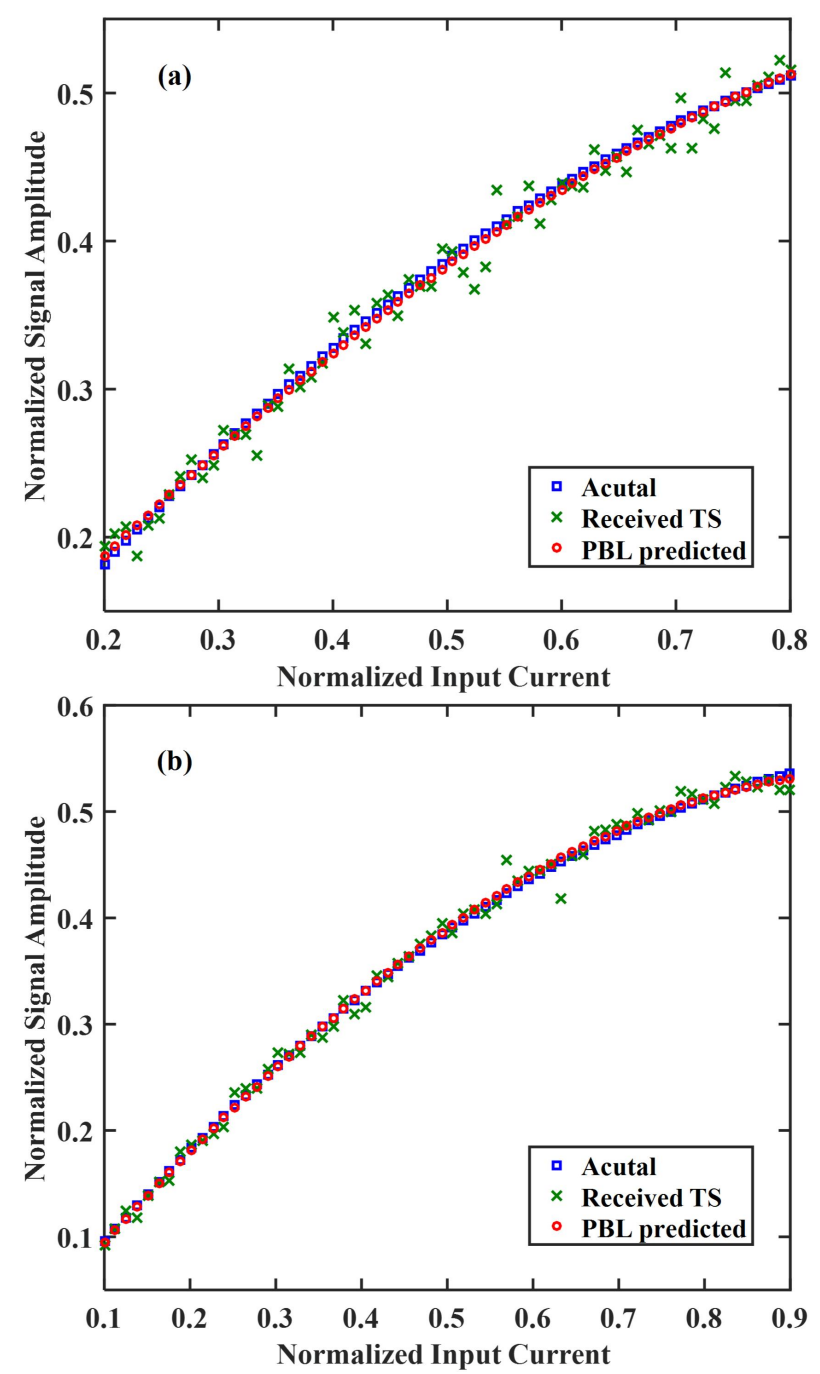

Figure 4. Normalized signal amplitude vs. normalized input current for (a) $\mathrm{MI}=0.6$ and (b) $\mathrm{MI}=0.8$.

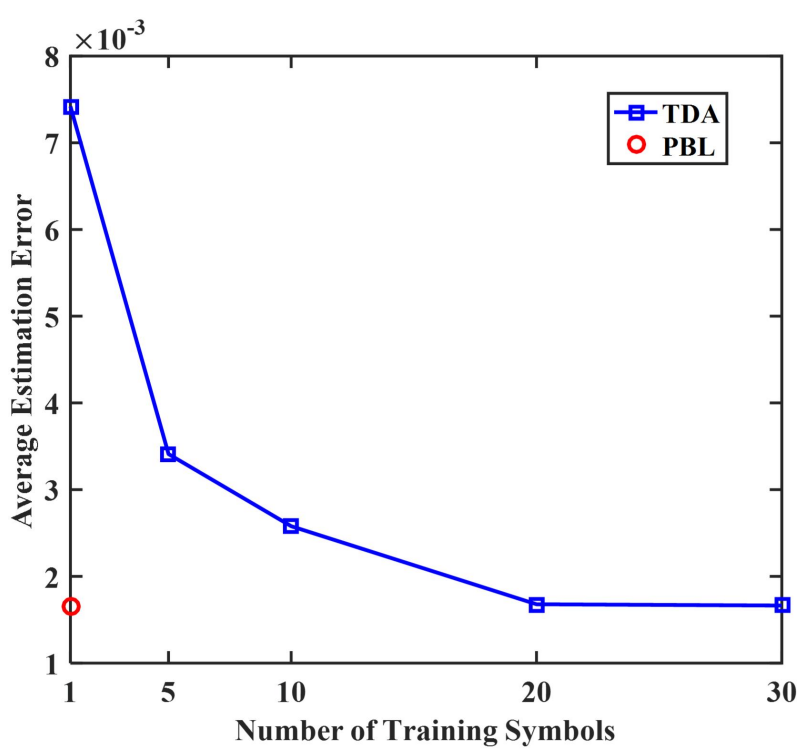

Figure 5. Average estimation error vs. number of training symbols.

The impact of the length of the TS on the BER performance of the OFDM-based nonlinear VLC system is also analyzed. Figure 6 shows the BER vs. the length of the TS for the conventional 
TDA scheme with different numbers of TSs and the proposed PBL-based scheme with a single TS, with $\mathrm{MI}=0.8$ and DI $=0.5$. Evidently, for both conventional TDA and the proposed PBL-based scheme, the BER performance is substantially improved with the increase of the length of the TS and stable BERs can be guaranteed when the length of the TS is about 64 .

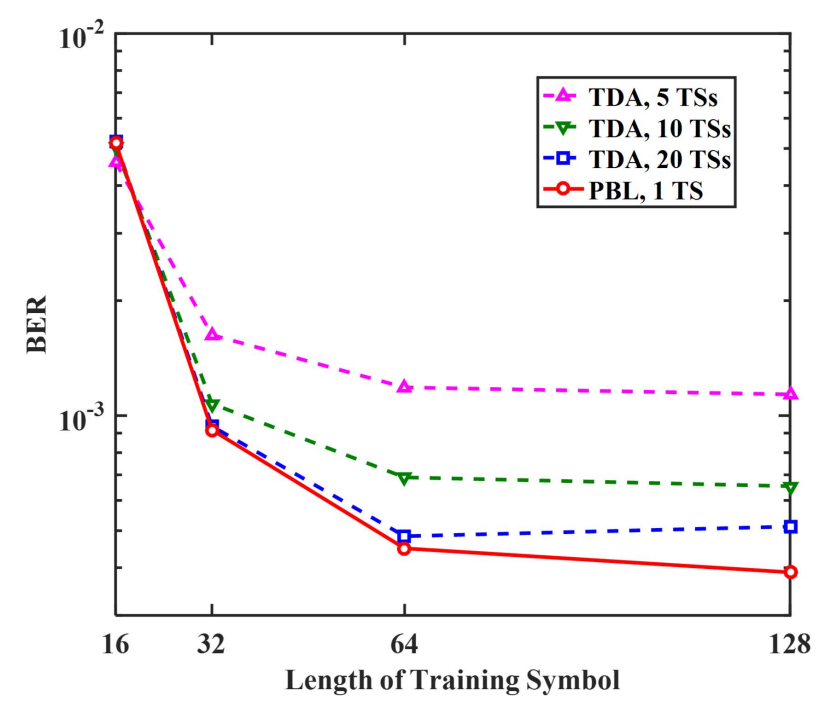

Figure 6. BER vs. length of training symbol.

Based on the analysis above, we evaluate and compare the BER performance vs. MI for the OFDM-based nonlinear VLC system without and with LED nonlinearity compensation, where DI $=0.5$ and the length of the TS is fixed at $N=64$. As shown in Figure 7, when no LED nonlinearity compensation is considered, the BER reduces with the increase of the MI and an MI of 0.87 is required to reach the BER threshold of $10^{-3}$. Moreover, the BER improvement becomes insignificant by further increasing MI when MI is larger than 0.9, suggesting the adverse effect of LED nonlinearity on the BER performance of the OFDM-based nonlinear VLC system. However, when the conventional TDA scheme is applied, the BER performance can be substantially improved. For TDA with 5 TSs, the required MI to reach BER $=10^{-3}$ is reduced to 0.80 . By further increasing the number of TSs, the BER performance can be further enhanced. In contrast, when the proposed PBL-based scheme with a single TS is adopted, the required MI to reach $\mathrm{BER}=10^{-3}$ is only about 0.77 . Furthermore, nearly the same BER performance can be achieved by the conventional TDA scheme with totally 20 TSs and the proposed PBL-based scheme with a single TS. Therefore, a substantial reduction of the training overhead for LED nonlinearity mitigation can be achieved by using the proposed PBL-based scheme in comparison to the conventional TDA scheme, which indicates a greatly improved overall spectral efficiency of the OFDM-based nonlinear VLC system. The corresponding 16QAM constellation diagrams are also shown as insets in Figure 7. 


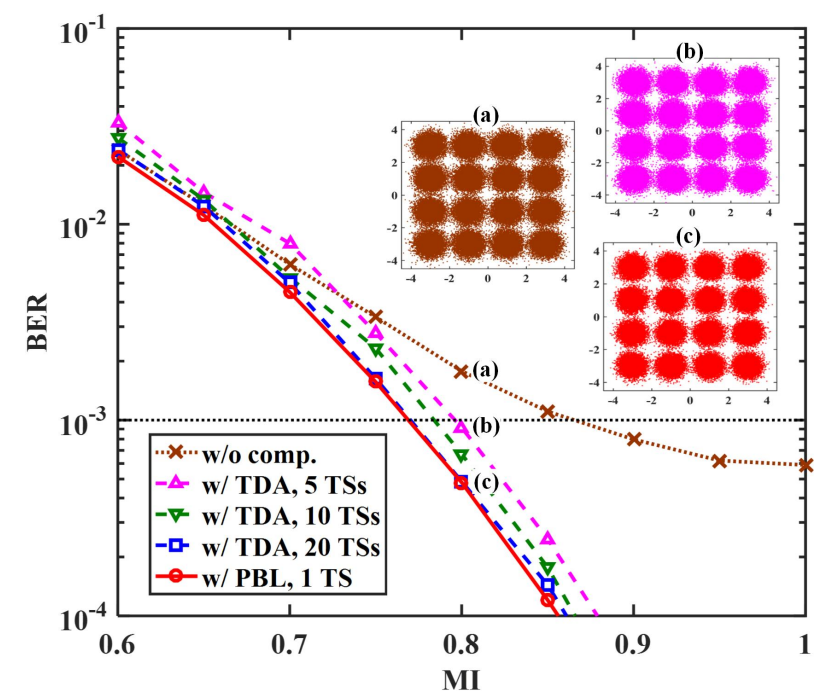

Figure 7. BER vs. MI without and with LED nonlinearity compensation.

Finally, we analyze the computational complexity of the proposed PBL-based LED nonlinearity estimation and compensation scheme. For the fast PBL regression model adopted here, PBL regression is initialized with the bias only and the basis functions are iteratively added, updated or deleted to increase the marginal likelihood [35]. Since adding basis functions requires most of the computations, the worst case is that a new basis function is added at each iteration and the worst-case computational complexity is given by $O\left(N_{i t} M^{2}\right)$ with $N_{i t}$ being the iteration number and $M$ being the number of basis functions. However, the worst case scenarios are highly impossible to occur due to the inherent sparsity of PBL regression. As introduced in [31], for common cases, an approximation of the computational complexity is about $O\left(N_{i t} N_{n z}^{2}\right)$ with $N_{n z}$ being the number of non-zero elements in the weight vector $w_{\mathrm{MP}}$. More specifically, by using the fast marginal likelihood maximization method, only three elements out of totally $M=65$ elements in $w_{\text {MP }}$ are non-zero, i.e., $N_{n z}=3$. Consequently, the proposed PBL-based LED nonlinearity estimation and compensation scheme is computational efficient which is suitable for the implementation in practical VLC systems with computing capability-limited user terminals.

\section{Conclusions}

In this paper, we have proposed a novel LED nonlinearity estimation and compensation scheme based on PBL regression for OFDM-based nonlinear VLC systems. The performance of the proposed PBL-based scheme has been evaluated by numerical simulations and further compared with the conventional TDA scheme. The obtained simulation results have shown that the proposed PBL-based scheme can accurately estimate the nonlinear power-current curve of the LED and hence efficiently compensate the adverse effect of LED nonlinearity. More specifically, the proposed PBL-based scheme with a single TS can achieve a comparable BER performance as the conventional TDA scheme with totally 20 TSs. Therefore, the required training overhead for LED nonlinearity mitigation can be substantially reduced and the overall system spectral efficiency can be greatly improved by adopting the proposed PBL-based scheme. It is also shown that the proposed PBL-based LED nonlinearity estimation and compensation scheme is computational efficient, which is suitable for potential application in practical VLC systems.

Author Contributions: Conceptualization, C.C., X.D., Y.Y. and L.Z.; Formal analysis, C.C., X.D., Y.Y. and H.Y.; Funding acquisition, C.C.; Project administration, C.C. and P.D.; Writing-original draft, C.C. and P.D.; Writing-review \& editing, C.C., X.D., Y.Y., P.D., H.Y. and L.Z.

Funding: This research was funded by the Starting Research Fund from the Chongqing University (No. 02140011044110). 
Conflicts of Interest: The authors declare no conflict of interest.

\section{References}

1. Komine, T.; Nakagawa, M. Fundamental analysis for visible-light communication system using LED lights. IEEE Trans. Consum. Electron. 2004, 50, 100-107. [CrossRef]

2. Jovicic, A.; Li, J.; Richardson, T. Visible light communication: Opportunities, challenges and the path to market. IEEE Commun. Mag. 2013, 51, 26-32. [CrossRef]

3. Pathak, P.H.; Feng, X.; Hu, P.; Mohapatra, P. Visible light communication, networking, and sensing: A survey, potential and challenges. IEEE Commun. Surv. Tutor. 2015, 17, 2047-2077. [CrossRef]

4. Wu, D.; Zhong, W.D.; Ghassemlooy, Z.; Chen, C. Short-range visible light ranging and detecting system using illumination light emitting diodes. IET Optoelectron. 2016, 10, 94-99. [CrossRef]

5. Deng, X.; Mardanikorani, S.; Wu, Y.; Arulandu, K.; Chen, B.; Khalid, A.M.; Linnartz, J.P.M. Mitigating LED nonlinearity to enhance visible light communications. IEEE Trans. Commun. 2018, 66, 5593-5607. [CrossRef]

6. Rajbhandari, S.; Chun, H.; Faulkner, G.; Cameron, K.; Jalajakumari, A.V.; Henderson, R.; Tsonev, D.; Ijaz, M.; Chen, Z.; Haas, H.; et al. High-speed integrated visible light communication system: Device constraints and design considerations. IEEE J. Sel. Areas Commun. 2015, 33, 1750-1757. [CrossRef]

7. Elgala, H.; Mesleh, R.; Haas, H.; Pricope, B. OFDM visible light wireless communication based on white LEDs. In Proceedings of the IEEE 65th Vehicular Technology Conference (VTC Spring), Dublin, Ireland, 22-25 April 2007; pp. 2185-2189.

8. Marshoud, H.; Kapinas, V.M.; Karagiannidis, G.K.; Muhaidat, S. Non-orthogonal multiple access for visible light communications. IEEE Photonics Technol. Lett. 2015, 28, 51-54. [CrossRef]

9. Chen, C.; Zhong, W.D.; Yang, H.; Du, P. On the performance of MIMO-NOMA-based visible light communication systems. IEEE Photonics Technol. Lett. 2017, 30, 307-310. [CrossRef]

10. Yang, Y.; Chen, C.; Zhang, W.; Deng, X.; Du, P.; Yang, H.; Zhong, W.D.; Chen, L. Secure and private NOMA VLC using OFDM with two-level chaotic encryption. Opt. Express 2018, 26, 34031-34042. [CrossRef]

11. Chen, C.; Zhong, W.D.; Yang, H.; Du, P.; Yang, Y. Flexible-rate SIC-free NOMA for downlink VLC based on constellation partitioning coding. IEEE Wirel. Commun. Lett. 2019, 8, 568-571. [CrossRef]

12. Zeng, L.; O’Brien, D.C.; Le Minh, H.; Faulkner, G.E.; Lee, K.; Jung, D.; Oh, Y.; Won, E.T. High data rate multiple input multiple output (MIMO) optical wireless communications using white LED lighting. IEEE J. Sel. Areas Commun. 2009, 27, 1654-1662. [CrossRef]

13. Chen, C.; Zhong, W.D.; Wu, D. On the coverage of multiple-input multiple-output visible light communications. IEEE/OSA J. Opt. Commun. Netw. 2017, 9, D31-D41. [CrossRef]

14. Chen, C.; Zhong, W.D.; Wu, D. Indoor OFDM visible light communications employing adaptive digital pre-frequency domain equalization. In Proceedings of the Conference on Lasers and Electro-Optics (CLEO), San Jose, CA, USA, 5-10 June 2016; paper JTh2A.118.

15. Le Minh, H.; O’Brien, D.; Faulkner, G.; Zeng, L.; Lee, K.; Jung, D.; Oh, Y.; Won, E.T. 100-Mb/s NRZ visible light communications using a postequalized white LED. IEEE Photonics Technol. Lett. 2009, 21, 1063-1065. [CrossRef]

16. Ying, K.; Yu, Z.; Baxley, R.J.; Qian, H.; Chang, G.K.; Zhou, G.T. Nonlinear distortion mitigation in visible light communications. IEEE Wirel. Commun. 2015, 22, 36-45. [CrossRef]

17. Stepniak, G.; Siuzdak, J.; Zwierko, P. Compensation of a VLC phosphorescent white LED nonlinearity by means of Volterra DFE. IEEE Photonics Technol. Lett. 2013, 25, 1597-1600. [CrossRef]

18. Mitra, R.; Bhatia, V. Chebyshev polynomial-based adaptive predistorter for nonlinear LED compensation in VLC. IEEE Photonics Technol. Lett. 2016, 28, 1053-1056. [CrossRef]

19. Liang, S.; Jiang, Z.; Qiao, L.; Lu, X.; Chi, N. Faster-than-Nyquist precoded CAP modulation visible light communication system based on nonlinear weighted look-up table predistortion. IEEE Photonics J. 2018, 10, 1-9. [CrossRef]

20. Lu, H.; Jin, J.; Wang, J. Alleviation of LED nonlinearity impact in visible light communication using companding and predistortion. IET Commun. 2019, 13, 818-821. [CrossRef]

21. Mitra, R.; Bhatia, V. Adaptive sparse dictionary-based kernel minimum symbol error rate post-distortion for nonlinear LEDs in visible light communications. IEEE Photonics J. 2016, 8, 1-13. [CrossRef] 
22. Deng, X.; Mardanikorani, S.; Arulandu, K.; Linnartz, J.P.M. Novel Post-distortion to Mitigate LED Nonlinearity in High-speed Visible Light Communications. In Proceedings of the IEEE Globecom Workshops (GC Wkshps), Abu Dhabi, United Arab Emirates, 9-13 December 2018; pp. 1-6.

23. Wang, Y.; Tao, L.; Huang, X.; Shi, J.; Chi, N. Enhanced performance of a high-speed WDM CAP64 VLC system employing Volterra series-based nonlinear equalizer. IEEE Photonics J. 2015, 7, 1-7. [CrossRef]

24. Lu, X.; Wang, K.; Qiao, L.; Zhou, W.; Wang, Y.; Chi, N. Nonlinear compensation of multi-CAP VLC system employing clustering algorithm based perception decision. IEEE Photonics J. 2017, 9, 1-9. [CrossRef]

25. Ma, J.; He, J.; Shi, J.; Zhou, Z.; Deng, R. Nonlinear Compensation Based on K-Means Clustering Algorithm for Nyquist PAM-4 VLC System. IEEE Photonics Technol. Lett. 2019, 31, 935-938. [CrossRef]

26. Lu, X.; Lu, C.; Yu, W.; Qiao, L.; Liang, S.; Lau, A.P.T.; Chi, N. Memory-controlled deep LSTM neural network post-equalizer used in high-speed PAM VLC system. Opt. Express 2019, 27, 7822-7833. [CrossRef] [PubMed]

27. Miao, P.; Zhu, B.; Qi, C.; Jin, Y.; Lin, C. A Model-Driven Deep Learning Method for LED Nonlinearity Mitigation in OFDM-based Optical Communications. IEEE Access 2019, 7, 71436-71446. [CrossRef]

28. Nguyen, T.; Mhatli, S.; Giacoumidis, E.; Van Compernolle, L.; Wuilpart, M.; Mégret, P. Fiber nonlinearity equalizer based on support vector classification for coherent optical OFDM. IEEE Photonics J. 2016, 8, 1-9. [CrossRef]

29. Lake, B.M.; Salakhutdinov, R.; Tenenbaum, J.B. Human-level concept learning through probabilistic program induction. Science 2015, 350, 1332-1338. [CrossRef]

30. Tipping, M.E. Sparse Bayesian learning and the relevance vector machine. J. Mach. Learn. Res. 2001, 1, 211-244.

31. Chen, C.; Zhong, W.D.; Zhao, L. Sparse Bayesian RVM regression based channel estimation for IM/DD OFDM-VLC systems with reduced training overhead. In Proceedings of the IEEE International Conference on Communications Workshops (ICC Workshops), Paris, France, 21-25 May 2017; pp. 162-167.

32. Zhao, L.; Wang, L.; Yang, L.; Zoubir, A.M.; Bi, G. The race to improve radar imagery: An overview of recent progress in statistical sparsity-based techniques. IEEE Signal Process. Mag. 2016, 33, 85-102. [CrossRef]

33. Zhao, L.; Wang, L.; Bi, G.; Zhang, L.; Zhang, H. Robust frequency-hopping spectrum estimation based on sparse Bayesian method. IEEE Trans. Wirel. Commun. 2014, 14, 781-793. [CrossRef]

34. Li, J.; Huang, Z.; Liu, X.; Ji, Y. Hybrid time-frequency domain equalization for LED nonlinearity mitigation in OFDM-based VLC systems. Opt. Express 2015, 23, 611-619. [CrossRef]

35. Tipping, M.E.; Faul, A.C. Fast marginal likelihood maximisation for sparse Bayesian models. In Proceedings of the 9th International Workshop on Artificial Intelligence and Statistics, Key West, FL, USA, 3-6 January 2003; pp. 1-13.

36. Aggarwal, P.; Kabra, T.; Ahmad, R.; Bohara, V.A.; Srivastava, A. Adaptive learning architecture-based predistorter for nonlinear VLC system. Photonics Netw. Commun. 2019, 1-12. [CrossRef] 